\title{
Efeitos de Imagens Estáticas com Diferentes Representações de Movimento sobre a Percepção Subjetiva de Tempo
}

\author{
Effect of Static Images with Different Representations of Movement Under \\ the Subjective Perception of Time
}

\author{
Francisco Carlos Nather* \& José Lino Oliveira Bueno \\ Universidade de São Paulo, Ribeirão Preto, Brasil
}

\begin{abstract}
Resumo
O estudo da percepção de movimento e de tempo subjetivo pode ser ampliado com a utilização de imagens como estímulos visuais. Este trabalho teve como objetivo verificar se duas imagens estáticas com diferentes representações de movimento afetariam distintamente a percepção temporal. Participantes universitários não treinados em artes visuais submetidos a imagens com diferentes sugestões de movimento reproduziram o tempo de apresentação dos estímulos sob o paradigma prospectivo. Os resultados mostraram que a fotografia com menor sugestão de movimento (Estímulo A) foi julgada mais curta que aquela com maior sugestão de movimento (Estímulo B), embora ambas tenham sido subestimadas em relação à duração real. A análise dos dados das escalas de diferencial semântico, referentes a 4 critérios de julgamento de movimento em imagens estáticas, mostrou que o Estímulo A foi julgado possuindo menos movimento que o Estímulo B. Conclui-se que a sugestão de movimento numa imagem estática alonga a experiência temporal.

Palavras-chave: tempo subjetivo; imagens; movimento
\end{abstract}

\begin{abstract}
The study of the perception of movement and subjective time can be expanded with the utilization of images as visual stimuli. The aim of this work was to verify if two static images with different representations of movement would affect distinctly temporal perception. University students not trained in visual arts submitted to images with different suggestions of movement reproduced the time of presentation of the stimuli under the prospective paradigm. The results showed that the picture with the lesser suggestion of movement (Stimulus A) was judged shorter than the one with more suggestion of movement (Stimulus B), although both have been underestimated in relation to real duration. The analysis of the data of semantic differential scales, referred to 4 criteria of movement of judgment in static images, showed that stimulus A was judged having less movement than stimulus B. The conclusion was that the suggestion of movement in a static image extends the temporal experience.

Keywords: subjective time; images; movement
\end{abstract}

O tempo é essencial para o ser humano se orientar a sua volta. A idéia de tempo se relaciona a estados de consciência, que se fundamentam na sucessão e duração dos fatos, indicando ser o tempo uma experiência pessoal interior, uma unidade intuitiva com duração homogênea (Bueno, 1985a). Estudos que procuram esclarecer como o tempo é percebido pelos humanos têm enfatizado o componente subjetivo na percepção temporal (Block \& Zakay, 1997; Fraisse, 1984; Zakay, 1990).

\footnotetext{
"Endereço para correspondência: Prof. Dr. José Lino O. Bueno /Francisco C. Nather. Faculdade de Filosofia, Ciências e Letras, Departamento de Psicologia e Educação, Universidade de São Paulo, Avenida dos Bandeirantes 3900, Ribeirão Preto, SP, 14040-901. Fone: 16 602-3697. Fax: 16 633-5668. E-mail: fnather@usp.br E-Mail: jlobuen@ffclrp.usp.br F. C. Nather recebeu uma bolsa de doutorado do CNPq; J. L O. Bueno recebeu uma bolsa de Pesquisador I e Auxílio à Pesquisa do CNPq.

Agradecimentos: À Thaíza H. Figueiredo pela colaboração nas análises estatísticas, Lézio S. Bueno Júnior pela colaboração na coleta de dados e Juliana C. Souza e João Luís S. Borin pelo auxílio técnico.
}

Experimentalmente, o tempo subjetivo pode ser analisado em termos de estimação que está baseada nas mudanças percebidas pelo sujeito (Fraisse, 1984). Segundo Zakay (1990), por exemplo, quanto maiores são as mudanças e o número de estímulos ocorridos por unidade de tempo, maior é a duração do evento para o indivíduo.

A duração tem sido empregada de diferentes maneiras, segundo os paradigmas de tempo subjetivo: o retrospectivo e o prospectivo. No paradigma retrospectivo o indivíduo não sabe que irá fazer uma estimação temporal, estando tal método relacionado à duração relembrada de algo que já aconteceu. No prospectivo, contrariamente, o indivíduo tem a informação de que serão feitas estimações temporais, o que envolve a duração de algo que está sendo vivenciado no momento presente. Implicitamente, nestas distintas maneiras de experienciar a duração, diferentes estratégias e características do intervalo de tempo podem ser utilizadas pelos indivíduos para estimar o tempo.

O padrão de experiências temporais e suas relações com os eventos do mundo objetivo dependem do contexto no qual estão inseridos (Bueno, 1985b). Em virtude disso, nas 
pesquisas de tempo subjetivo, a natureza da estimulação, as características do intervalo de tempo analisado, bem como a sua intensidade e modalidade envolvem diferentes processamentos perceptivos temporais (Zakay \& Block, 2004), que podem resultar em alterações nas estimações temporais para mais (superestimações) ou para menos (subestimações) (Block, 1990).

Os estudos sobre estimação de tempo subjetivo têm empregado principalmente estímulos acústicos, gerando avanços na compreensão do processo subjacente à estimação subjetiva de tempo. Bueno, Firmino e Engelman (2002) utilizando dois trechos de músicas clássicas, uma simples e outra complexa, encontraram que a mais complexa foi julgada mais longa que a mais simples, embora ambas possuíssem mesma duração temporal. A utilização desses estímulos musicais, que ocorrem num espaço de tempo, tem evidenciado que distintas características das composições musicais podem ser utilizadas como marcadores temporais pelos indivíduos. A manipulação experimental desses marcadores como, as escalas musicais (Ramos \& Bueno, 2004), as modulações de trechos musicais distantes e próximos (Firmino \& Bueno, 2002), variações tonais nos finais musicais (Lelis, 2002) e o contexto rítmico (Jones \& Boltz, 1989) mostraram que distintos processos participam da percepção temporal.

Os principais modelos de tempo subjetivo propostos têm concordado que fatores psicológicos associados à quantidade de informação contida nos estímulos estejam envolvidos no processo de estimação temporal - haveria uma relação direta entre o tempo de estimação e os processos de memória e atenção. O modelo do Armazenamento (Ornstein, 1969) propõe que intervalos de tempo iguais parecerão ter diferentes durações porque um é mais complexo e necessita de mais esforço para ser armazenado na memória. O modelo da Atenção (Thomas \& Weaver, 1975) enfoca que a duração estimada depende da quantidade total de esforço de atenção requerido por um dado estímulo - isto é, estímulos complexos parecem mais longos, porque requerem uma atenção seletiva maior. O modelo da Mudança Cognitiva (Block, 1985) supõe que a duração estimada aumenta com o número de mudanças contextuais do organismo ou da situação do meio ambiente. Exemplificando, mudanças de estratégias de processamento da informação do estímulo ou mudanças nas demandas de tarefa podem afetar a estimação temporal de um evento. O modelo do Contraste (Boltz, 1989) supõe que estimativas de durações avaliadas são determinadas pela estrutura dos eventos a serem julgados. Eventos que permitem futura atenção orientada, através de uma previsibilidade estrutural, são julgados em termos dos seus finais: se eles confirmam ou violam as expectativas.

A percepção visual de movimento tem sido estudada em seus diversos aspectos. Aymoz e Viviani (2004), Bedell, Chung, Ogmen e Patel (2003), Viviani e Aymoz (2001) salientam o tempo de percepção de estímulos em movimento. Grondin (2003) aponta mecanismos de processamento temporal entre eventos visuais. Kellman (1995) aborda a ontogênese da percepção de espaço e mo- vimento. Ehrenstein (2003) enfatiza algumas condições básicas no ver o movi-mento real, movimento aparente e a extrapolação de movimentos, que envolve aspectos da percepção temporal.

A percepção temporal já foi utilizada em estudos que procuravam compreender a percepção de movimento em imagens (Alderson \& Whiting, 1974; Hellmann, 1996; Peterken, Brown \& Bowman, 1991), provavelmente porque o movimento só existe dentro de um espaço e tempo determinados. Entretanto, nestas e em outras pesquisas de percepção de movimento, os participantes estimavam o tempo de chegada de um estímulo que estava percorrendo uma trajetória a uma velocidade determinada, mas que desaparecia em determinado instante (Nather \& Bueno, 2005). Na verdade, o tempo de chegada é a medida de um intervalo de tempo, compreendendo o desaparecimento do estímulo visual juntamente com a projeção de tempo necessário para o estímulo chegar à posição final da sua trajetória experimental.

Segundo Nather e Bueno (2005), os dados da literatura de tempo subjetivo têm indicado que os estímulos visuais em movimento são julgados mais longos que os estímulos parados. Entretanto, em dois diferentes trabalhos que utilizaram formas geométricas iguais, um no paradigma prospectivo (Brown, 1995) e outro no retrospectivo (Predebon, 2002b), não foi encontrada concordância de resultados. No paradigma prospectivo os estímulos em movimento foram superestimados em relação ao tempo real de estimulação e, além disso, os movimentos mais rápidos tenderam a alongar a duração temporal em maior magnitude que os movimentos mais lentos. Ao contrário, no retrospectivo, os estímulos mais rápidos foram subestimados em relação aos mais lentos.

O tempo total de exposição também pode afetar a eficiência da estimação temporal. Segundo Brown (1995), em estímulos visuais, os episódios curtos tendem a ser superestimados e os longos subestimados na presença ou na ausência de um movimento real. Isto sugere que as marcações temporais existentes nos estímulos visuais que se movem (velocidade, orientação, distância, etc.) podem ser usadas como pontos de referência e funcionar como marcações temporais em estímulos visuais estáticos.

As distintas representações de movimentos paralisados em imagens estáticas figurativas ilustram alguns destes marcadores. Análises de inúmeras pinturas, desenhos e fotografias de períodos históricos distintos (Cutting, 2002) mostraram que o movimento vem sendo representado de diferentes maneiras: por meio das diferentes posições entre as partes do corpo humano e suas vestimentas (equilíbrio dinâmico); com a exibição de imagens estroboscópicas de um objeto numa mesma imagem; pela desfocalização de partes da imagem; por meio da inclinação de um objeto em movimento; com o uso de setas vetoriais na imagem que indicam um movimento que já aconteceu. Braddick (1995) considera que a sugestão de movimento representado numa imagem pode ocorrer quando está sugerida a trajetória do objeto; quando são reconhecidas a forma e textura cinética do objeto representado, bem como a sua 
estrutura tridimensional em movimento; e em função das relações espaciais entre as figuras da imagem.

A eficiência desses recursos de representação pode ser verificada segundo 4 critérios, que dizem que a imagem deve evocar sensação de movimento, representar claramente o objeto em movimento, indicar a direção e indicar a intensidade deste movimento (Cutting, 2002). Tais critérios podem ou não existir conjuntamente numa mesma imagem ou obra de arte.

Berlyne (1974) propõe uma Estética Experimental que permite a análise da apreciação de obras de arte segundo critérios experimentais. Esta proposta considera as propriedades colativas das obras de arte de duas maneiras diferentes: por meio da abordagem sintética, que consiste em selecionar partes, variáveis ou fatores particulares de um trabalho artístico; ou por meio da abordagem analítica, que utiliza obras de arte genuínas. A abordagem analítica claramente aproxima o investigador do que acontece quando as pessoas se defrontam com a arte na vida real, apesar de ser mais difícil ao experimentador controlar as variáveis independentes operantes ou mesmo medi-las.

Imagens estáticas e imagens em movimento têm revelado importantes aspectos da percepção temporal em humanos. As distintas características e marcações temporais destes diferentes tipos de estímulos podem gerar variados graus de percepção, que podem promover alterações na percepção de tempo. A literatura especializada tem examinado relações entre tempo subjetivo e imagens estáticas e em movimento (Brown, 1995), mas não de imagens estáticas com representação de movimento.

No presente estudo foram usadas fotografias de objetos artísticos, conforme a abordagem sintética de Berlyne (1974), para verificar se o maior ou o menor movimento representados numa imagem estática afetariam a estimação subjetiva de tempo. A representação de movimento de imagens estáticas foi caracterizada segundo os critérios propostos por Braddick (1995) e Cutting (2002).

\section{Método}

\section{Participantes}

Participaram do experimento 30 estudantes universitários não treinados em artes visuais (12 homens e $18 \mathrm{mu}$ lheres), com idades entre 18 e 38 anos, que relataram apresentar visão normal. O experimento foi conduzido segundo as normas do Comitê de Ética (Resolução nº 196/96).

\section{Equipamento e Material}

O experimento foi realizado durante o período diurno numa instituição universitária da cidade de Ribeirão Preto, numa sala experimental branca com as luzes apagadas. Encostada em uma das paredes havia uma mesa retangular e uma cadeira para os participantes, e à sua esquerda estava posicionada a cadeira do experimentador. Sobre a mesa havia um teclado de computador modificado conectado a um microcomputador Notebook HP-Pavilion ZE5375. O teclado modificado possuía todas as suas te- clas cobertas com papel camurça branco com exceção de três teclas distintamente marcadas, que possuíam as funções de iniciar a apresentação das imagens e registrar as reproduções temporais dos participantes.

O programa Wave Surfer foi usado para a apresentação dos estímulos e para o registro das estimações temporais. Um caderno de dados experimentais continha as escalas de representação de movimento adaptadas dos 4 critérios de julgamento de movimento propostos por Cutting (2002), que dizem que há maior ou menor sugestão de movimento numa imagem se: (a) O objeto ou figura é capaz de evocar sensação de movimento; (b) A representação do objeto é bem definida; (c) O movimento é intenso; (d) A direção do movimento é bem definida. As escalas de diferencial semântico de representação de movimento eram do tipo Likert de sete pontos que, apresentadas a quatro participantes universitários num piloto, mostraram-se adequadas para o experimento.

Foram utilizados como estímulos fotografias de dois objetos tridimensionais da série O Espelho produzida por Nather $(2004)^{1}$. A fotografia com menor sugestão de movimento foi a de uma boneca na posição sentada (Estímulo A), e a com maior sugestão de movimento, a de uma boneca na posição em pé sugerindo o movimento de uma dança de balé (Estímulo B) ${ }^{2}$. O tempo total de apresentação de cada estímulo foi 36 segundos.

Foram feitas imagens fotográficas desses objetos tridimensionais em ângulos com maior e menor sugestão de movimento. As imagens utilizadas possuíam os seguintes critérios de representação de movimento: equilíbrio dinâmico, desfocalização de partes da imagem e inclinação ou deformação de partes da imagem do objeto descrevendo a direção do seu movimento (Cutting, 2002). Além destes, também estavam presentes: sugestão da trajetória do objeto, reconhecimento da forma cinética e informação da estrutura tridimensional do objeto em movimento (no caso de fotografias são a própria imagem fotografada) (Braddick, 1995).

Para definir quais estímulos seriam utilizados no experimento foram analisadas, individualmente, as fotografias de quatro bonecas que, segundo a avaliação do experimentador, representavam movimentos em diferentes intensidades. Essas imagens foram submetidas a quatro juízes com nível universitário (dois homens e duas mulheres), que julgaram verbalmente o seu movimento na forma de pontuações ( $\mathrm{O}$ a 10 pontos). Foram escolhidas as fotografias das bonecas 
com menor e maior pontuações médias: 4,31 para a boneca na posição sentada e 9,43 para a boneca em pé.

As fotografias foram feitas com um fundo infinito branco que contrastava com as cores ligeiramente rosadas das bonecas. As fotografias foram realizadas com uma máquina fotográfica digital Sony Cyber-Shot DSC7 7 posicionada numa mesma distância para as duas bonecas, o que manteve os objetos focalizados no centro das imagens e com tamanhos semelhantes. As imagens digitais passaram por um tratamento visual no programa Adobe Photoshop 7.0 para ajustes de luminosidade e de saturação e favorecer imagens fiéis dos objetos originais.

O tamanho final das imagens na tela do Notebook foi $14 \times 18 \mathrm{~cm}$ na resolução de $1024 \times 768$ dpi. As imagens ocuparam a posição central do monitor do computador sendo o restante da tela preenchido com a cor branca.

\section{Procedimento}

Cada um dos 30 participantes foi individualmente conduzido à sala e convidado a sentar na sua cadeira. Em seguida foram explicadas verbalmente as tarefas que ele deveria realizar durante o experimento: "Você observará duas imagens diferentes e após o término de cada imagem reproduzirá duração temporal de cada uma utilizando o teclado de computador à sua frente. Para isso acontecer, após a apresentação da primeira imagem, você apertará a tecla marcada iniciar e começará a sua contagem de tempo. Quando você achar que o tempo que passou foi igual àquele de apresentação da imagem que você observou, você apertará a tecla marcada finalizar e isso irá concluir a sua estimação temporal. Para a segunda imagem você irá repetir o mesmo procedimento". A seguir, o participante era informado que durante as suas duas estimações temporais ele não observaria qualquer imagem, pois a tela do computador ficaria em branco. Logo após o término das estimações, o participante era orientado a observar novamente cada imagem e a preencher as escalas de diferencial semântico de representação de movimento.

Todos os participantes foram posicionados de maneira a estarem com suas cabeças voltadas à região central da tela do Notebook numa distância fixa de $50 \mathrm{~cm}$ da tela, com a orientação de prestar atenção às imagens. Antes do começo das tarefas, possíveis dúvidas para a realização do experimento foram esclarecidas.
As imagens foram apresentadas em duas seqüências diferentes para dois grupos de participantes: Estímulo A Estímulo B (Seqüência A-B, Grupo I, N=16 participantes) e Estímulo B - Estímulo A (Seqüência B-A, Grupo II, $\mathrm{N}=14$ participantes). Além disso, os participantes de cada grupo foram divididos em dois subgrupos iguais, onde um era orientado a prestar a atenção no movimento representado nas imagens (Grupo Movimento) e o outro não (Grupo Sem Movimento). Assim, para o Grupo Movimento, no início das explicações das tarefas dos participantes era dito: "Você observará duas imagens diferentes que representam movimentos em diferentes intensidades. Após o término de cada imagem você reproduzirá duração temporal de cada uma utilizando o teclado de computador à sua frente...”. O restante das instruções foi igual ao do Grupo Sem Movimento.

Terminadas essas tarefas era realizada a entrevista. As respostas dos participantes a um questionário de treze questões referentes a dados pessoais, possíveis experiências artísticas do participante e dados referentes ao experimento foram anotadas pelo experimentador. Uma das questões colheu os julgamentos verbais do tempo de duração dos estímulos e outra procurou investigar a possibilidade deles despertarem emoções ou sentimentos. Havia também um espaço para preenchimento de observações finais identificadas pelo experimentador, como dúvidas ou falhas durante a execução do experimento pelos participantes.

\section{Resultados}

Foram calculados, para cada um dos estímulos, os valores médios das estimações temporais e das pontuações das escalas de diferencial semântico. Todos os dados foram submetidos a análises de variância (ANOVA) e, em alguns casos, foram realizadas análises post hoc utilizando o teste Newman-Keuls. Em todas as análises foi considerado o nível de significância $\mathrm{p} \leq 0,05$.

A análise dos valores médios das reproduções temporais de todos os participantes revelou que ocorreram subestimações temporais para os dois estímulos utilizados: 31,57 segundos para o Estímulo A e 34,13 segundos para o Estímulo B. As estimações temporais médias para o Estímulo A foram inferiores àquelas do Estímulo B (Tabela 1).

Tabela 1

Valores Médios e Desvios Padrão em Segundos das Reproduções Temporais dos Participantes

\begin{tabular}{llll}
\hline Sequiências & Estímulo A & Estímulo B & $\mathrm{N}=$ \\
\hline Sequiência A-B & $29,76( \pm 7,01)$ & $32,74( \pm 5,07)$ & 16 \\
Sequiência B-A & $33,63( \pm 4,36)$ & $35,73( \pm 5,77)$ & 14 \\
Sequiências A-B e B-A & $31,57( \pm 6,15)$ & $34,13( \pm 3,41)$ & 30 \\
\hline
\end{tabular}


A análise estatística ANOVA comparando os fatores seqüências (A-B e B-A) e estímulos (A e B) mostrou diferença significativa apenas para o fator estímulos $[\mathrm{F}(1,28)=4,14$; $\mathrm{p}=0,04]$, indicando não ter ocorrido efeito de apresentação de estímulos. Isto mostra que na média das duas seqüências apresentadas aos participantes o Estímulo A foi estimado com menor duração que o Estímulo B, apesar de ambos possuírem a mesma duração de 36 segundos. Na Tabela 1 estão representados os valores médios das estimações temporais e os seus respectivos desvios padrão.

Quando foram comparados os fatores subgrupos Grupo Movimento (GM) e Grupo Sem Movimento (GSM) e estímulos (A e B) não foi verificada diferença significa-tiva entre os grupos de participantes que foram e que não foram informados ou induzidos verbalmente a prestar atenção no

Tabela 2 movimento representado nas imagens $[\mathrm{F}(1,28)=0,30$; $p=0,58]$. Foram encontradas diferenças estatísticas significativas nas estimações temporais entre os estímulos A e B $[\mathrm{F}(1,28)=4,22 ; \mathrm{p}=0,05]$ na média dos subgrupos.

As análises de variância ANOVA tendo como fatores os 4 critérios de julgamento de movimento e os Estímulos A e B mostraram diferenças significativas entre os julgamentos dos participantes nos 4 critérios $[\mathrm{F}(4,120)=34,4 \mathrm{O} ; \mathrm{p}=0,00]$, nos estímulos A e B $[\mathrm{F}(1,30)=52,89 ; \mathrm{p}=0.00]$ e nos estímulos dependendo das perguntas $[\mathrm{F}(4,120)=13,39 ; \mathrm{p}=0,00]$. Análises post hoc mostraram diferenças significativas entre os estímulos A e B somente nos critérios 1, 3 e 4 . Isto indica que os participantes apresentaram pontuações maiores para o Estímulo B nas respostas que envolviam representação de movimento (Tabela 2).

Pontuações Médias e Desvios Padrão das Respostas dos Participantes aos Critérios de Julgamento de Movimento

\begin{tabular}{llc}
\hline Critérios & Estímulo A & Estímulo B \\
\hline 1. O objeto é capaz de evocar sensação de movimento? & $2,45( \pm 1,54)$ & $5,09( \pm 1,68)$ \\
2. A representação do objeto é clara? & $4,87( \pm 1,92)$ & $5,61( \pm 1,40)$ \\
3. O movimento é intenso? & $2,06( \pm 1,48)$ & $4,29( \pm 1,71)$ \\
4. A direção do movimento é clara? & $2,51( \pm 1,80)$ & $5,12( \pm 1,85)$ \\
\hline
\end{tabular}

Os valores médios das respostas às perguntas números 1,3 e 4 foram superiores para o Estímulo B em comparação com o Estímulo A. Isto sugere que a imagem da boneca na posição sentada (Estímulo A) representou para os participantes menos movimento que a imagem da boneca na posição em pé em movimento de dança de balé (Estímulo B). O valor médio da resposta número 2 foi semelhante para os dois estímulos.

A análise conjunta dos dados das reproduções temporais e das escalas de diferencial semântico indicou haver relação entre a representação de movimento em imagens estáticas e a experiência de passagem de tempo. Tal resultado pode ser verificado quando se observam os valores das respostas às perguntas $O$ objeto é capaz de evocar sensação de movimento?, O movimento é intenso?, A direção do movimento é clara? que, possuindo maior indicação de atenção ao movimento, mostraram valores de estimações temporais maiores para o Estímulo B que para o Estímulo A.

Apesar da análise dos julgamentos verbais ter concordado com a análise das reproduções temporais, no sentido de o Estímulo A ser mais curto que o Estímulo B, não foram verificadas diferenças estatísticas nas estimações temporais verbais tendo como fatores seqüências (A-B e B-A), subgrupos (GM e GSM) e estímulos (A e B). As médias das estimações temporais verbais dos participantes para os estímulos A e B foram respectivamente $38,05 \mathrm{~s}$ e $40,46 \mathrm{~s}$, indicando superestimações temporais em relação ao tempo real de estimulação de 36s.

As entrevistas realizadas posteriormente à coleta de dados revelaram que a maioria dos participantes $(70 \%)$ relatou ter usado o recurso de contar mentalmente o tempo de exposição das imagens para realizar as suas estimações temporais. Quando foram analisados apenas esses participantes que contaram o tempo, observou-se que $85 \%$ deles estimaram diferentemente a duração dos Estímulos A e B. A maioria desses participantes, cerca de 65\%, julgou que o Estímulo A possuía uma duração menor que o Estímulo B, mostrando que mesmo contando os participantes estimaram diferentemente os estímulos.

Mais de $60 \%$ dos participantes relataram que as imagens não despertaram qualquer sentimento ou emoção. Embora a pergunta fosse a respeito de emoções e sentimentos, alguns participantes disseram que as imagens fizeram lembrar a infância ou alguma outra época das suas vidas. Uma pequena porcentagem dos participantes informou que as imagens evocaram neles sentimentos de alegria, felicidade e tranqüilidade.

\section{Discussão}

A análise dos dados das reproduções temporais e dos julgamentos verbais mostrou que a sugestão de movimento numa imagem estática figurativa alterou a percepção subjetiva de tempo. Nas reproduções temporais o estímulo sem sugestão de movimento (Estímulo A) foi subestimado em relação àquele com sugestão de movimento (Estímulo B). Embora os julgamentos verbais não tenham apresentado resultados estatísticos diferentes, os dados sugerem uma tendência de os participantes subestimarem temporalmente o Estímulo A em relação ao Estímulo B. 
Os valores das reproduções temporais de 31,6s para o Estímulo A e de 34,1s para o Estímulo B foram menores que o tempo de 36s de exposição (subestimações temporais). Inversamente, nos julgamentos verbais os participantes tenderam a superestimar as durações dos estímulos. A possível explicação para as diferenças de estimação entre os métodos da reprodução e do julgamento verbal deve relacionar-se aos diferentes processos cognitivos que atuam nestas distintas maneiras de processar o tempo. Essa não concordância de resultados foi também descrita em outros estudos que utilizaram diferentes métodos de estimação temporal (Eisler, 2003; Lelis, 2002). Brown (1995), em um de seus experimentos utilizando o método da produção, encontrou valores inversos daqueles onde foi usado o método da reprodução: na produção é solicitado ao participante que estime um intervalo de tempo pré-determinado, e na reprodução ele estima um intervalo de tempo experienciado.

Estimações temporais distintas podem ser obtidas também em estudos que utilizaram estímulos iguais, porém empregando os paradigmas retrospectivo e prospectivo (Brown, 1995; Predebon 2002a, 2002b). Em estudos de tempo subjetivo devem existir diferentes processos cognitivos atuantes (Zakay \& Block, 2004), que podem ser evidenciados em função do contexto no qual os experimentos são realizados. Isto também sugere que diferentes processos podem ser estudados utilizando um mesmo paradigma temporal.

No presente estudo, que utilizou o paradigma prospectivo, cerca de $70 \%$ dos participantes relatou ter contado ou elaborado algum tipo de contagem mental para fazer as estimações temporais. Considerando apenas os participantes que contaram, verificou-se que mesmo na contagem as estimações temporais dos Estímulos A e B foram diferentes para a maioria dos participantes (70\%), sendo novamente o Estímulo A subestimado em relação ao Estímulo B. Tal resultado confirma a idéia de que no paradigma prospectivo a atenção dos indivíduos orienta-se ao componente temporal, muito embora a relação estabelecida entre estímulo e indivíduo possa sobrepujá-la, algo que poderia ser explicado pelo modelo atencional de tempo subjetivo (Thomas \& Weaver, 1975). Segundo este modelo, a duração estimada depende da quantidade total de esforço de atenção requerido por um dado estímulo: os mais complexos podem parecer mais longos porque requerem maior atenção seletiva.

Aparentemente os resultados das estimações temporais aproximaram-se mais daqueles de estudos que utilizaram estímulos em movimento no paradigma prospectivo, nos quais imagens estáticas foram subestimadas em relação a imagens em movimento (Brown, 1995). De fato, a imagem estática sem sugestão de movimento foi subestimada em relação àquela com sugestão de movimento, mesmo quando foi informado aos participantes que eles observariam imagens com representação de movimento em diferentes intensidades. Provavelmente, a percepção de um movimento sugerido numa imagem e a visualização de um movimento real devam estar relacionadas a um processamento percep- tivo semelhante. Neste sentido, seria interessante verificar se estímulos com diferentes sugestões de intensidade de movimento suscitariam estimações temporais proporcionais, como aquelas encontradas na literatura: aumentos na velocidade de um estímulo causam maiores aumentos nas estimações temporais (Brown, 1995; Predebon, 2002b).

É necessário considerar o que nas fotografias pode ter causado as alterações nos julgamentos temporais. As principais diferenças entre as imagens fotográficas eram as posições das partes do corpo das duas bonecas, que sugeriam maior ou menor movimento. As análises das pontuações dos julgamentos verbais dos 4 critérios de representação de movimento indicaram que o Estímulo A foi considerado com menos movimento que o Estímulo B em três das quatro perguntas: O objeto é capaz de evocar sensação de movimento?, O movimento é intenso? e A direção do movimento é clara, sugerindo que os participantes foram capazes de relatar diferentes intensidades de movimento entre as duas imagens. O fato de as pontuações para a outra pergunta, A representação do objeto é clara?, não terem sido muito diferentes entre os estímulos não contradiz este dado. Provavelmente, isso pode ser devido ao conteúdo desta pergunta não ser diretamente voltado a alguma sugestão de movimento, mas à fidelidade de representação do objeto numa imagem bidimensional. Uma vez que foram utilizadas fotografias de objetos reais, eram esperadas pontuações mais aproximadas dos estímulos para este critério de julgamento de movimento.

Outro resultado contribuiu com a idéia de que foi o movimento na imagem da boneca na posição de movimento de balé (Estímulo B) como o responsável pelas diferenças nos julgamentos temporais entre o Estímulo A (boneca na posição sentada) e o Estímulo B: 60\% dos participantes não relataram qualquer sentimento ou emoção ao observar as imagens. Dos $40 \%$ restantes, metade não mencionou sentimentos e emoções, mas lembranças de uma época passada (infância); os demais citaram principalmente sentimentos positivos de alegria e felicidade.

Possivelmente, a ligação das imagens há algum tempo passado possa também se relacionar à representação de movimento equilíbrio dinâmico (Cutting, 2002) existente nas imagens apresentadas. Tal representação, que se refere às assimetrias causadas pelas posições entre os membros do corpo e vestimentas, é rapidamente percebida como em movimento também em razão da sua familiaridade. Chamada de narrativa, a disposição diferencial de objetos e pessoas em esculturas foi muito utilizada por artistas plásticos que objetivavam dar a um objeto estático tridimensional uma dimensão temporal (ver Krauss, 1977/2001).

A verdade é que as assimetrias fazem com que os olhos movimentem-se mais rapidamente, propiciando maior exploração do campo de visão (Cutting, 2002). Assim, a imagem com maior sugestão de movimento poderia ser mais complexa que a outra porque, independentemente das suas outras propriedades colativas, ela apresenta maiores pontos de referência para os olhos. Isso quer dizer que esta imagem possuía pontos de movimento mais evidentes, ou um maior número de marcadores temporais. 
Quanto maior o número de pontos de referência indicando e remetendo a uma maior sugestão de movimento, mais complexa pode ser considerada a imagem e, conseqüentemente, maior é o armazenamento e memória necessários para processá-la. Assim, a superestimação temporal da imagem com mais movimento poderia ser explicada segundo o modelo de tempo subjetivo do armazenamento (Ornstein, 1969).

Como encontrar um caminho indicativo numa obra bidimensional, um começo e um fim, ou para onde ir com os olhos em qualquer ponto ou instante numa imagem se ela é captada como um todo? (Arnheim, 1974/1980). Segundo o autor existem caminhos vetoriais preferenciais por onde os olhos passam sem a percepção consciente do observador, ou seja, numa abordagem gestáltica, mesmo não possuindo representação de movimento, uma obra visual abstrata ou figurativa possui mais ou menos movimento em decorrência da disposição dos seus elementos constituintes. Portanto, uma obra figurativa de movimento apresentaria índices de movimentos maiores, por existirem representações (marcadores) verdadeiras da direção, trajetória ou velocidade de algum movimento paralisado na imagem. A sugestão de movimento poderia também evocar lembranças de movimento por induzir a elaboração de algo já conhecido que está para acontecer. Tal fato foi observado em algumas das respostas dos participantes que disseram que a imagem da boneca na posição de movimento de balé os remeteu a um movimento que estaria por vir. Talvez, outros resultados seriam obtidos se fosse utilizada uma imagem de um movimento que acabou de acontecer.

Para Cupchick e Gebotys (1988), a duração de um episódio estético determinaria quanto tempo um observador gasta em atividades cognitivas e na compreensão de uma obra de arte. A experiência da duração variaria em razão direta com o processamento perceptivo da complexidade de um estímulo, ou seja, quanto maior o número de imagens ou mudanças, mais lentamente o tempo levaria a passar, ocasionando superestimação temporal. Analisando diferentes propriedades colativas como a definição, clareza e complexidade de pinturas impressionistas genuínas (abordagem analítica; Berlyne, 1974), estes autores verificaram que indivíduos não treinados em artes visuais superestimaram o tempo nos seus julgamentos verbais, enquanto que os participantes treinados, não. Embora no presente estudo tenham sido utilizadas partes de uma obra de arte (abordagem sintética), com o intuito de analisar a representação de movimento, foram também observadas superestimações temporais em participantes não treinados, possivelmente em virtude de o movimento suscitar maior trabalho cognitivo.

\section{Referências}

Alderson, G. J. K., \& Whiting, H.T. A. (1974). Prediction of linear motion. Human Factors, 16, 495-502.

Arnheim R. (1980). Arte e percepção visual: Uma psicologia da visão criadora (I. T. de Faria, Trad.). São Paulo, SP: Pioneira. (Original publicado em 1974).
Aymoz, C., \& Viviani, P. (2004). Perceptual asynchronies for biological and non-biological visual events. Vision Research, 44, 1547-1563.

Bedell, H. E., Chung, S. T. L., Ogmen, H., \& Patel, S. S. (2003). Color and motion: Which is the tortoise and which is the hare? Vision Research, 43, 2403-2412.

Berlyne, D. E. (1974). The new experimental aesthetics. In D. E Berlyne (Ed.), Studies in the new experimental aesthetics (pp. 125). Toronto, Canada: University of Toronto.

Block, R. A. (1985). Contextual coding in memory: Studies of remembered duration. In J. A. Michon \& J. L. Jackson (Eds.), Time, mind and behavior (pp. 169-178). Berlin, Germany: Springer-Verlag.

Block, R. A. (1990). Models of psychological time. In R. A. Block (Ed.), Cognitive Models of Phychological Time. (pp. 1-35). Hillsdale, NJ: Lea Publishers.

Block, R. A., \& Zakay, D. (1997). Prospective and retrospective duration judgments: A meta-analytic review. Psychonomic Bulletin Review, 4(2), 184-197.

Boltz, M. (1989). Time judgments of musical endings: Effects of expectancies on the "filled interval effect". Perception \& Psychophsics, 46(5), 409-418.

Braddick, O. (1995). The many faces of motion perception. In O. Braddick (Ed.), The artful eye (pp. 205-231). Oxford, UK: Oxford University Press.

Brown, S. W. (1995). Time, change, and motion: The effects of stimulus movement on temporal perception. Perception \& Psychophysics, 1, 105-116.

Bueno, J. L. O. (1985a). A questão do tempo [Resumo]. In A Jacquemin (Ed.), Anais, XV Reunião Anual de Psicologia (pp. 196-197). Ribeirão Preto, SP: SBP.

Bueno, J. L. O. (1985b). Tempo biológico, psicológico e espaçosocial [Resumo]. In A. Jacquemin. (Ed.), Anais, XV Reunião Anual de Psicologia (pp. 198-206). Ribeirão Preto, SP: SBP.

Bueno, J. L. O., Firmino, E. A., \& Engelman, A. (2002). Influence of generalized complexity of a musical event on subjetive time estimulation. Perceptual and Motor Skills, 94, 541-547.

Cupchick, G. C., \& Gebotys, R. (1988). The experience of time, pleasure, and interest during aesthetic episodes. Empirical Studies of the Arts, 6(1), 1-12.

Cutting, J. E. (2002). Representing motion in a static image: Constraints and parallels in art, science, and popular culture. Perception, 31(10), 1165-1193.

Ehrenstein, W. (2003). Basics of seeing motion. Arquivos Brasileiros de Oftalmologia, 66, 44-52.

Eisler, A. D. (2003). The human sense of time: Biological, cognitive and cultural considerations. In R. Buccheri, M. Saniga \& W. M. Stuckey (Eds.), The nature of time: Geometry, Physics and perception. (pp. 5-18). London: Kluwer Academic.

Firmino, E. A., \& Bueno, J. L. O. (2002). Modulação tonal e tempo subjetivo [Resumo]. In Sociedade Brasileira de Psicologia (Ed.), Anais, XXXII Reunião Anual de Psicologia (pp. 154). Florianópolis, SC: SBP.

Fraisse, P. (1984). Perception and estimation of time. Annual Review of Psychology, 35, 1-36.

Grondin, S. (2003). Processing time between visual events. Arquivos Brasileiros de Oftalmologia, 66, 96-104.

Hellmann, A. (1996). Studies on the perception and extrapolation of motion. In A. Hellmann (Ed.), Auditory perception of approaching sound sources, and time-of-arrival judgements (pp. 28-33). Bochum, Germany: Universidade de Bochum.

Jones, M. R., \& Boltz, M. (1989). Dynamic attending and responses to time. Psychological Review, 96, 459-491. 
Kellman, P. J. (1995). Ontogenesis of space and motion perception. In W. Epstein \& S. Rogers (Ed.), Perception of space and motion (pp. 327-364). Madison, WI: Madison Academic Press.

Krauss, R. E. (2001). Caminhos da escultura moderna (J. Fischer, Trad.). São Paulo, SP: Martins Fontes. (Original publicado em 1977).

Lelis, C. M. C. (2002). Influência de audições musicais com variação de composição sobre estimação objetiva de tempo. Disser tação de Mestrado não-publicada, Curso de Pós-Graduação em Psicologia e Educação, Universidade de São Paulo, Ribeirão Preto, SP.

Nather, F. C., \& Bueno, J. L. O. Tempo subjetivo e percepção de movimento em obras de arte. Manuscript submitted for publication.

Ornstein, R. E. (1969). On the experience of time. Baltimore: Penguin.

Peterken, C., Brown, B., \& Bowman, K. (1991). Predicting the future position of a moving target. Perception, 20, 5-16.

Predebon, J. (2002a). Stimulus motion and retrospective time judgements. Acta Phychologica, 109, 213-225.

Predebon, J. (2002b). Viewing distance and estimates of duration. Perceptual and Motor Skills, 95, 326-328.

Ramos, D., \& Bueno, J. L. O. (2004). Fatores afetivos na música modal influenciam a estimação subjetiva de tempo [Resumo]. In Sociedade Brasileira de Psicologia (Ed.), Anais, XXXIV Reunião Anual de Psicologia (p. 81). Ribeirão Preto, SP: SBP.

Thomas, E. A. C., \& Weaver, W. B. (1975). Cognitive processing and time perception. Perception \& Psychophysics, 17, 363-367.

Viviani, P., \& Aymoz., C. (2001). Colour, form, and movement are not perceived simultaneously. Vision Research, 41, 2909-2918.

Zakay, D. (1990). The evasive art of subjective time measurement: Some methodological dilemas. In R. A. Block (Ed.), Cognitive models of psychological time (pp. 59-84). Hillsdale, NJ: Erlbaum.

Zakay, D., \& Block, R. A. (2004). Prospective and retrospective duration judgments: An executive-control perspective. Acta Neurobiologiae Experimentalis, 64, 319-328. 